\title{
Unwanted tooth movement produced by the bonded retention wire : the "wire syndrome," observations, theories, clinical consequences : second part
}

\author{
F. Roussarie ${ }^{1}$, G. Douady ${ }^{2}$ \\ 1 Orthodontist - Qualified Specialist, Private Practice Bourg-la-Reine \\ 2 Computer Engineer
}

ABSTRACT

Sometimes teeth can move even if they seem to have been perfectly stabilised by a retention wire. We call this phenomenon the "wire syndrome."

It has been well described both in the mandibular and maxillary arches in part one of this article.

For the moment, there has been no mechanical explanation for this phenomenon in the scientific literature. After an analysis of photographic documents from fellow specialists and an examination of cases from our own office, we have developed a theory to explain the mechanical element involved.

We will divide our analysis into two hypotheses. They could be inter-connected.

The first so-called "intrinsic" hypothesis groups together procedural erreurs which all lead to the use of an active bonded wire. In such cases, the wire is directly responsible for the movements. Its action is the same as that of an orthodontic device directly bonded on the teeth.

The second "extrinsic" hypothesis shows that a tooth with a well bonded wire can rotate if the link between the wire and the composite is broken and if force is present.

The clinical evidence we have gathered could allow us to combat this phenomenon efficiently.

\section{KEYWORDS}

Bonded retention, wire syndrome, unwanted versions, twisted wire, relapse, complication

\section{INTRODUCTION: A REMINDER OF THE DEFINITION OF "WIRE SYNDROME" AND ITS PREVALENCE}

In a previous article, we used the name "wire syndrome" to describe a whole range of situations in which one or several teeth connected to each other by a bonded wire present an unwanted version movement. This movement is mainly observed on the canines, but can also affect one or more incisors.

\section{Address for correspondence:}

Florence Roussarie - 77 boulevard Joffre - 92340 Bourg-La-Reine - France 


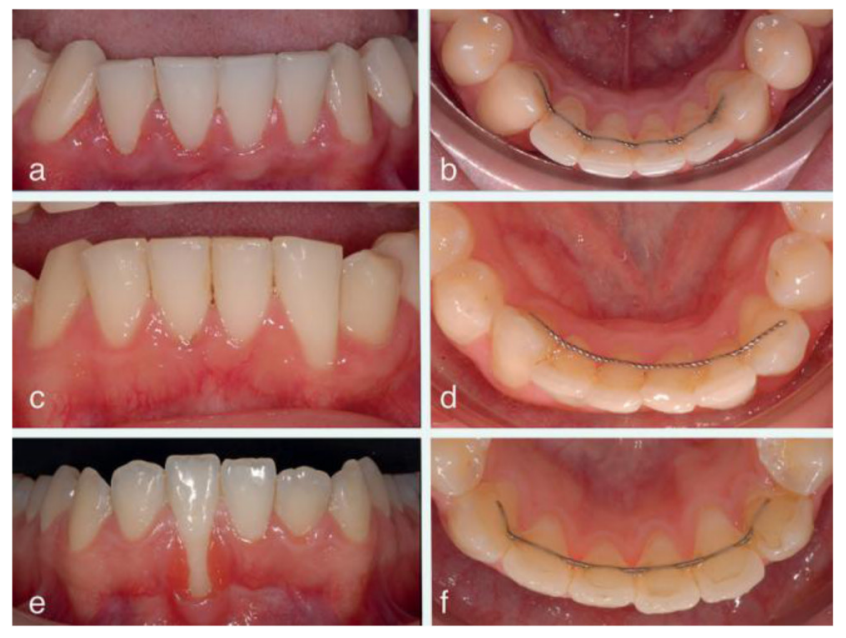

Figure 1

Typical form of "wire syndrome." $a, b$ : Coronolingual version of $43 ; b, c$ : Coronolingual version of 43 and coronovestibular version of 33; $d$, e: Radiculobuccal version of 41 .

The most common forms (Fig. 1) have been described in the first part of this article ${ }^{10}$.

The prevalence of this syndrome is still poorly studied. The most comprehensive study is by a Czech team ${ }^{3}$ on a cohort of 3,500 consecutive patients at the same practice treated by a single operator and monitored from 2008 to 2013. The authors found that 38 of the patients exhibited "wire syndrome." The wires used were 6-strand coaxial twisted steel wires, i.e., with a diameter of $0.0175^{\prime \prime}$ or $0.0215^{\prime \prime}$. The percentage of affected patients was $1.08 \%$. In a 2016 study by Wolf et al. ${ }^{13}$, casts were scanned and surimposed at the beginning, the end of treatment and after 6 months. They tracked movements found an extremely high percentage of severe cases (13\%) and moderate cases (30\%) 6 months after fitting the wire!

\section{DISCUSSION OF THE REASONS FOR THESE UNWANTED VERSION MOVEMENTS AND HYPOTHESIS DEVELOPMENT}

The force required to move a tooth is very small, especially if the duration of force application is very long, which is the case for bonded wires as they are left in place for several years.

Studies on the subject go back to Reitan $^{8}$, and indicate a necessary force of $5 \mathrm{~N} / \mathrm{mm}$ or $500 \mathrm{~g} / \mathrm{mm}$. More recently, Sifakakis' team ${ }^{12}$ argued that a force of
$1 \mathrm{~N} / \mathrm{mm}$ is more than enough to mobilize a tooth if the application of force is very long.

We distinguish two main groups of hypotheses:

1) Intrinsic hypothesis: the bonded or rebonded wire is active and exerts a force on the teeth. 
2) Extrinsic hypothesis: the bonded wire is passive, but the teeth connected to the wire move under the influence of an external force.

\section{Intrinsic hypothesis: active wire}

There are three possibilities to consider:

1. The bonded wire is active and exerts a force on the teeth.

2. The wire is deformed after bonding by external pressure and "activated" during this deformation.

3. An active wire is rebonded during the period of contention.

All of these situations support the fact that a very similar effect can be achieved, regardless of the direct or indirect bonding technique and the nature of the wire used.

What can make a retention wire active?

\section{The wire is active when bonded \\ We will separate the reflection into two parts depending on the bonding technique: direct or indirect.}

\section{- Indirect bonding technique}

For the wire to be effectively passive, it is necessary to control each step of the production line.

- Movement of the teeth between the arch impression and wire bonding

Movement is possible if an active chain has been left in place or if the patient has lost an attachment between the impression and device removal appointments. We suggest minimizing the time between the impression and bonding appointments. The ideal situation would be to take the impression and attach the retention wire on the same day ${ }^{5}$ to limit these risks as much as possible.

\section{- Lack of impression accuracy}

If the impression is conventionally made with alginate, it is important to choose an alginate with maximal flexibility because the impression will most often be taken in the presence of brackets. There can easily be a "pull" or distortion of the impression: the alginates recommended by orthodontic manufacturers are generally not the most suitable for this type of impression (class B). We suggest choosing an alginate with a makeup similar to those recommended for prostheses (class A) ${ }^{1}$.

On the other hand, digital impression is perfect because the presence of brackets does not affect impression accuracy.

- Mistakes during impression casting

Whatever the material used (plaster or silicone), if the casting does not exactly reproduce the model, the bonded wire will be active. It is therefore recommended to cast the alginate impression in a maximum of $45 \mathrm{~min}^{1}$ for maximum accuracy.

This is then comparable to a silicon impression. In addition, dimensional variations - due to water or room temperature —will be reduced if this time frame is maintained.

This is difficult to achieve if this action is delegated to a laboratory. It is therefore advisable to cast the impression in the office. When using a silicone material ${ }^{1}$, the setting, which is then extremely 
fast-around 3 min-makes it possible to stay within this time frame.

If a digital impression is taken, the 3D printer will offer a precise model, with a timescale chosen by the practitioner and compatible with the laboratory steps required for making the passive wire.

\section{- Wire production defects}

The wire should be bent beyond its elastic limit. It is better to use pliers than the fingers to shape the wire. The bend must be sharp enough to cross the elastic limit, otherwise the wire will exert a restoring force to resume its shape (Fig. 2).

- Excessive pressure in the mouth during bonding

The practitioner exerts too much or asymmetric pressure on the transfer tray, thus inducing wire deformation.
This pushes the teeth and causes movements.

As a solution to this difficulty, we propose carrying out an indirect bonding as we can see the wire directly: the pads of the lateral incisors are extended by temporary repositioning clips, which allow the verification of the non active force and adaptation of the wire before bonding 9 (Fig. 3).

\section{- Direct bonding technique}

The wire is deformed either in the chair by successive tests on the patient or on a model, but is bonded directly without transfer.

- Use of elastics or silk thread to keep the wire firmly applied on the lingual surface of the teeth

These thread-holding techniques induce a force lower than the elastic limit

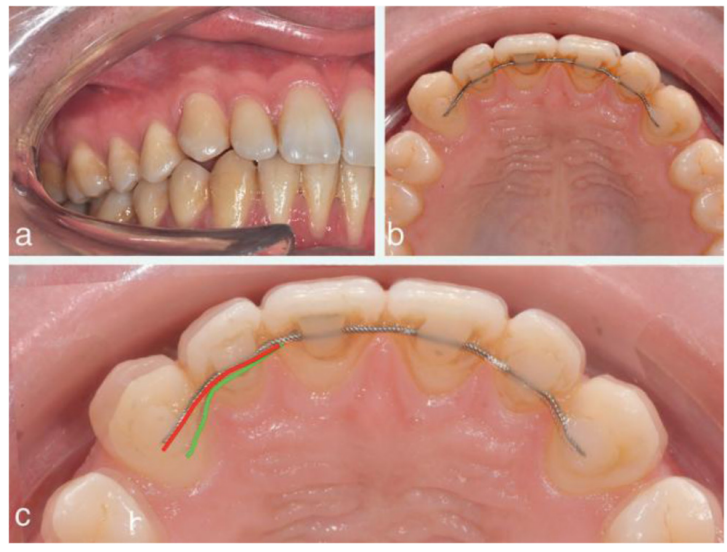

Figure 2

a) Twelve months after the wire is fitted, the right canine has a pronounced coronovestibular version.

b) The superposition, in 2 photos (end of treatment and one year later), clearly shows a modification of the wire shape.

It appears folded-green wire-during installation and straight-red wire-during consultation

1 year after wire bonding. The wire was bonded with force, with no plastic deformation between 12 and 13, and caused the canine movement. This type of deformation is necessarily visible in the few months following bonding. 

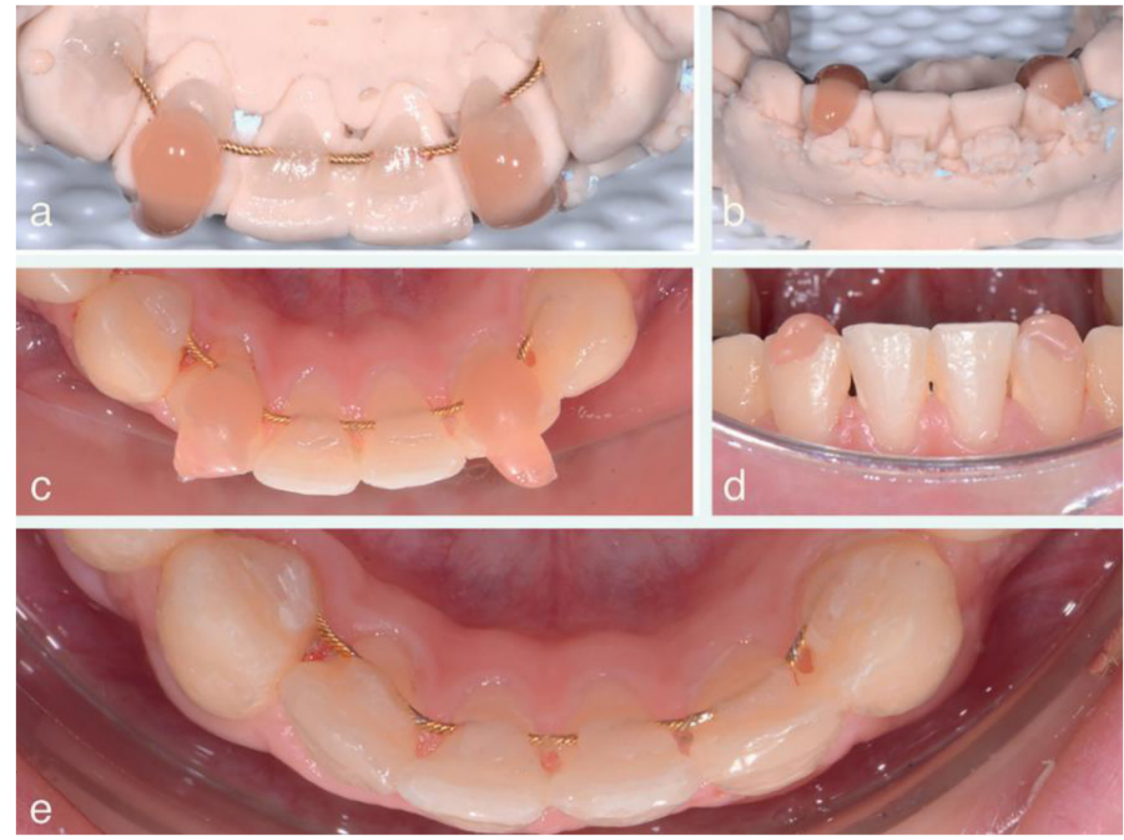

Figure 3

Indirect bonding in direct vision. $a, b$ : Wire preparation and composite studs on a silicone model with temporary positioning clips. $c, d$ : Wire bonded in place. e: The folds are cut and the composite pads on 32 and 42 are repolished.

and may be responsible for the deformation of the wire.

- Forced application of the wire during adhesive polymerization: when bonding in the mouth, the wire should not be forced using an instrument to better follow the anatomical contours of the lingual surfaces as closely as possible, but rather it should be bonded passively without any pressure ${ }^{14}$.

2. The wire is deformed by the interposition of a hard body

It has been described that the forces exerted by a male subject during occlusion can be up to $250 \mathrm{~N}$. The average forces observed during incision are 113 $\mathrm{N}$. These very strong forces can then cause irreversible deformations of the retention wire ${ }^{4}$.
However, it seems unlikely that the effects of random deformation created by occlusion forces can have the shape reproducibility seen in the syndrome described above.

This hypothesis mainly concerns the maxillary wires, which are more exposed than the mandibular wires to the forces generated during chewing. The wire may have been deformedfor example by a cherry or olive pitwithout coming off and thus initiating movement on the teeth.

\section{The rebonded wire is active}

In Figure 4, a retention wire was initially bonded from 13 to 23, then cut on 13 and 23, then again detached and bonded back to 12, probably in an active way, by bending the wire to bring it closer to the lingual surface. 


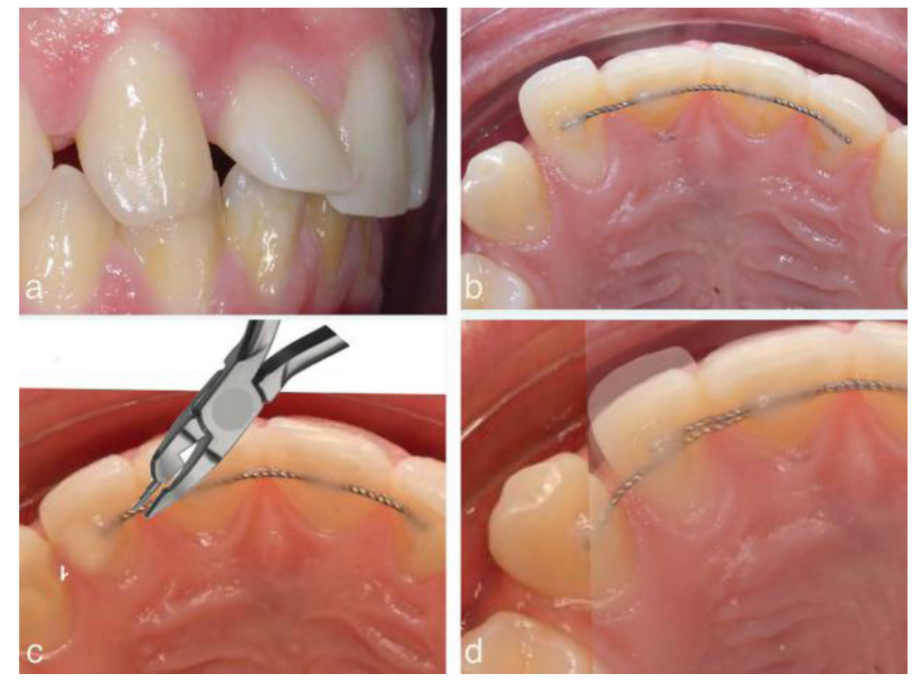

Figure 4

$a, b$ : Vestibuloversion of 12 after rebonding. $b$ : The wire is detached at a distance of 12 from the lingual surface. c: Pliers can be used to straighten it to establish contact with the enamel.

If the bend is too strong, which is difficult to evaluate because the wire is in contact with the tooth, the wire presses on the tooth and generates an uncontrolled version movement. $d$ : Superposition of the initial wire and the wire after rebonding with activation and the effect of vestibuloversion of the tooth.

However, these "intrinsic hypotheses" cannot explain the phenomenon of the "wire syndrome." This is important for three reasons:

1. The wire would become active immediately after bonding. Many patients, whose testimony we have been able to gather, explain that the movement began several years after bonding with a rapid evolution over several months leading up to the consultation.

2. Many factors are likely to cause a wire to activate, yet the observed effects take very reproducible forms.

3. The wire keeps its initial shape in majority of cases, which is in contradiction to the active wire hypothesis.

\section{Extrinsic hypothesis}

The teeth connected to the wire would move, without the wire becoming detached from the tooth, under the influence of an external force. The adhesive would fail at the wire-adhesive bonding point, which explains tooth movement while the wire remains firmly bonded to the lingual surface.

What are the forces involved in relapse?

The main force is "position memory," which justifies the use of a bonded wire.

This force depends on the initial situation, but also on the quality of finishes.

Wolf et al. ${ }^{13}$ in 2016 pointed out that the appearance of "wire syndrome" is due to the direct influence of a 
possible modification of the inter-canine distance and the amplitude of the overbite correction. The study was performed on a sample of 30 patients, treated consecutively with a vestibular multi-attachment. Using CBCT images taken before treatment, at the end of treatment, and 6 months after the contention, the author found $13.5 \%$ of severe forms of root movement at the level of the incisors and canines and $30 \%$ of moderate forms. Only 56.68\% are totally stable! The authors have succeeded in correlating these results with the increase in inter-canine distance and decrease in overbite. They concluded on the superiority of totally individualized devices, particularly in lingual technique. The fact that they respect the intercanine distance perfectly, make it possible to reduce the incidence of "wire syndrome" close to $0 \%$.

The maximum chewing force is $113 \mathrm{~N}$ at the incisors ${ }^{4}$. Some adults and children with onychophagia can exercise this level of strength. By comparison, note that the force of the digital clamp is $80.4 \mathrm{~N}$ and that of the total hand is $504.2 \mathrm{~N}^{11,12}$.

On the other hand, the force exerted by the tongue is around $400 \mathrm{~g}^{2}$, which we have seen is sufficient to induce tooth movement over a long time, but insufficient to deform a retention wire $^{12}$. On the other hand, if at the level of a tooth, the wire is separated from its composite stud, such a force acting on the crown is sufficient to move the tooth.

\section{What are our observations?}

We made observations on photographic documents of 115 cases at our practice (58) and other cases referred by orthodontists (57) that we have been collecting since 2008 :

- The methods of wire bonding studied are direct or indirect.

- The bonded wires are all round in section, twisted, with a diameter of 0.0175-0.0215" and comprising three wires or six coaxial wires.

- The composite studs are made of a photo-polymerizable filled composite.

Observation 1: The wire keeps its original shape. In 26 before/after cases in our possession-in which we can superimpose the photo from the day of wire bonding and the day of discovery of the "wire syndrome" - the wire retained its original shape in 24 patients (Fig. 5).

Observation 2: The affected teeth are moved with a high intensity. The roots can come out of the cortex. It does not resemble a relapse as we usually observe it. A simple relapse cannot take the teeth out of the cortex (Fig. 6).

Movements do not reproduce the initial malposition and cannot be confused with relapse (Fig. 7). Indeed, the observed movement is very constant,

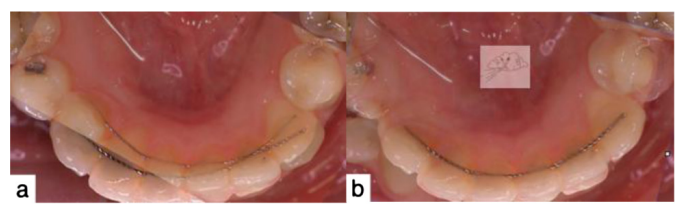

Figure 5

a) Premolar overlay of the photos taken on the day of bonding and the day of discovery of "wire syndrome." b: Superimposition of the two wires highlighting the fact that the wire has not changed shape and has not unfolded or folded. 


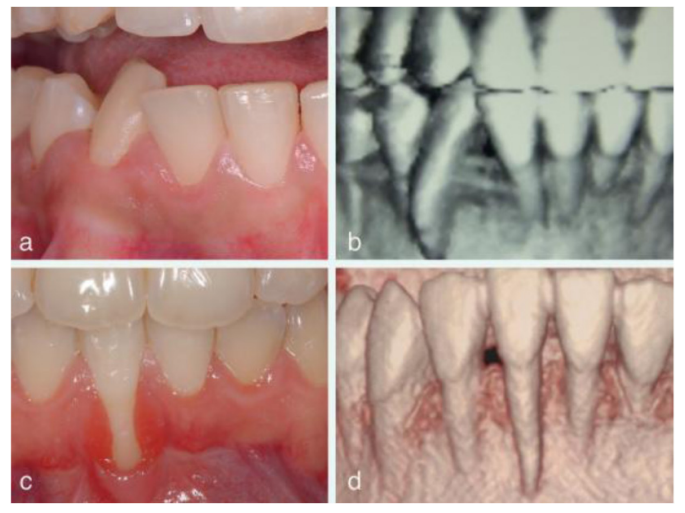

Figure 6

Comparison of clinical images and the section with CTCB reconstitution of two "wire syndromes." a, b: "Wire syndrome" affecting 43. c, d: "Wire syndrome" affecting 41.
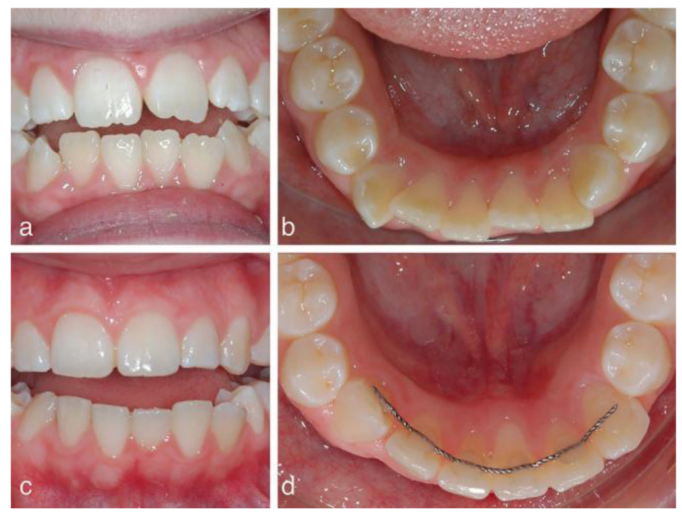

Figure 7

$a, b: 2004$, before orthodontic treatment.

c, d: 2011, 7 years after orthodontic treatment on the day of discovery of "wire syndrome": the canines are twisted in the opposite direction to their original position.

whereas a relapse would vary according to the initial malocclusion.

Observation 3: This movement stops as soon as the wire is removed, and the roots partially reposition in the cortex if occlusion allows
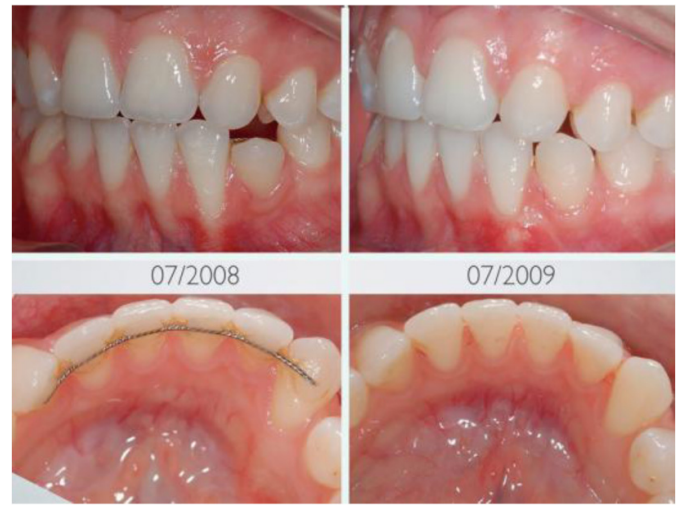

07/2009

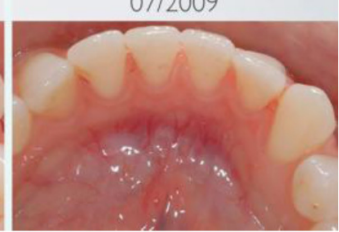

Figure 8

Partial but natural repositioning of the canine (33) and lateral incisor (32) 1 year after the removal of the retention wire.

it (Fig. 8). The periodontium, which is painful, improves with tooth repositioning.

Observation 4: The anterior joint is sometimes disturbed with an incisive occlusion from end to end up to the reversal of the anterior joint.

The incisive area is projected forward in all observed cases (Fig. 9). The force responsible for the movement therefore has a posterior-anterior component.

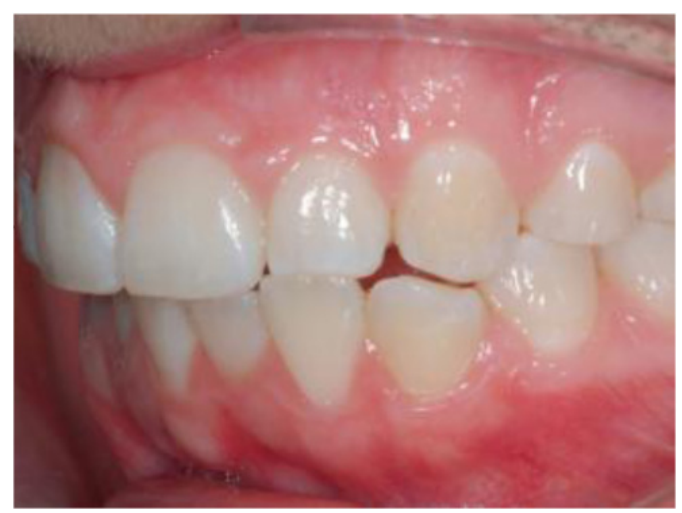

Figure 9

Anterior projection of the incisal area. 

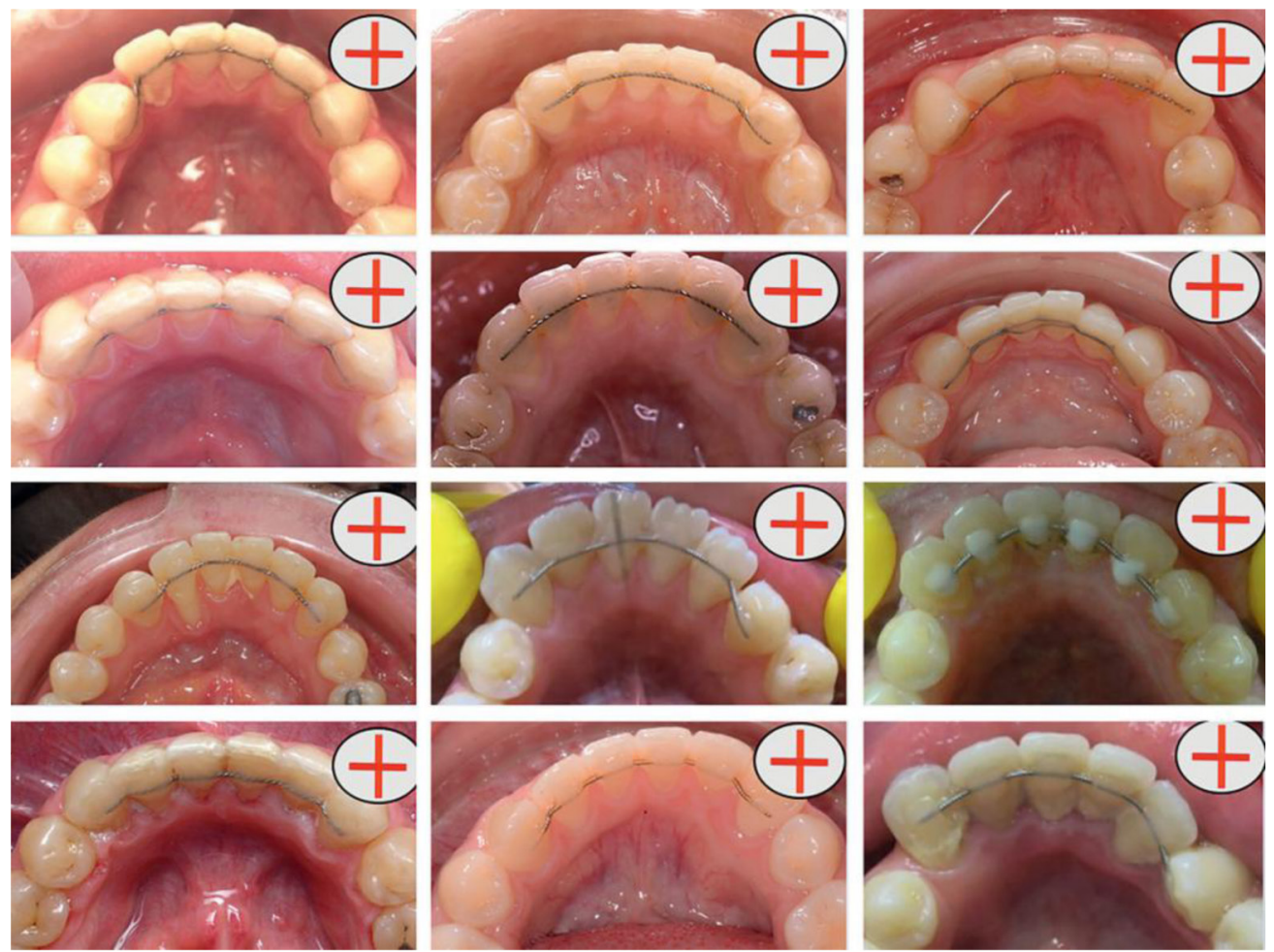

Figure 10

Sample of photographic documents, from referring orthodontists, on which we could observe the direction of rotation of the canines with respect to the winding direction of the twisted wires. They all turn in the opposite direction to the winding of the strands.

Observation 5: The direction of rotation observed on the canines moved, with respect to the turns of the wire, is always in the opposite direction to the rotational direction of the wires (Fig. 10).

We remind you that our observations are only on twisted wires.

This observation eliminates the hypothesis regarding the "non-twisted" wire (this would be an intrinsic cause), which is nevertheless the first intuitive hypothesis. This hypothesis is regularly mentioned by all authors.

Observation 6: "Wire syndrome" often starts several years after wire bonding, but suddenly shows a rapid evolution felt by the patient over the course of a few months.
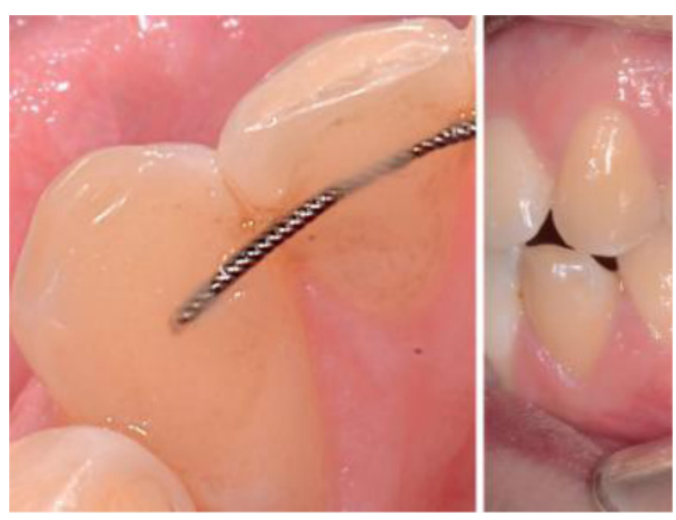

Figure 11

The canine rotates in the coronovestibular direction following the winding direction of the turns in the mesio-distal direction.

It seems to retreat on the wire. If the force was generated by the wire, it would turn in the opposite direction. 


\section{Explanation}

All these observations lead us to the development of a theory:

If the following occur simultaneously:

- a break in the adhesive at the adhesive-wire junction.

- application of an external force, the tooth is then rotated around the wire.

a) Breaking of the adhesive at the adhesive-wire junction

The tooth is connected to the wire via the bonded stud. If the bonded structure breaks at the adhesive-wire junction and the stud remains firmly bonded to the enamel, the tooth cannot-unless it fractures the entire structure-slide along the wire, which remains maintained in the bonded stud. This indicates a "helical link."

Indeed, when the wire is twisted, the adhesive and the tooth follow the shape of the wire and turn as if on a "screw thread" (Fig. 12).

b) Application of an external force If the tooth is subjected to an external force of the lingual impulse

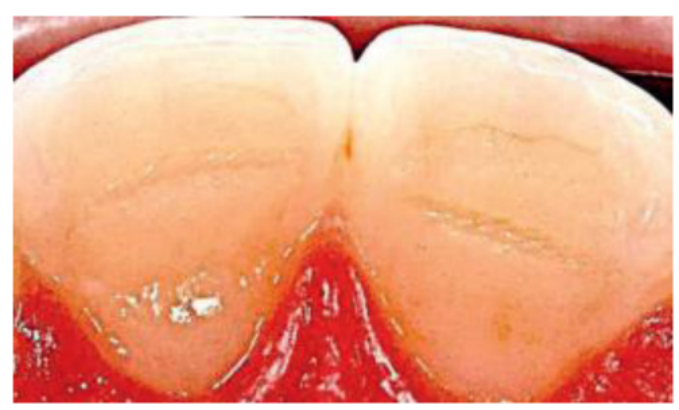

Figure 12

After removal by "tearing" of the bonded wire, we observe the imprint of the turns in the adhesive creating a real "screw thread." or parafunction type, the tooth/stud structure will rotate around the twisted wire in one direction or the other depending on the winding of the wire as if it were becoming "unscrewed." Sliding lateral movement is not possible because of the coils embedded in the adhesive.

The wire does not rotate, but remains static in the sense of a "solid bond" with the other teeth.

On the other hand, the adhesivewire bond gives way and the tooth can then move along the wire of the adhesive. The tooth "unscrews" and therefore marks an angle with the remaining bonded teeth.

The most frequent observation is in the canine (s); it is more rarely observed in an incisor.

According to the winding direction of the coils, the canine is oriented in the coronobuccal or coronolingual direction (Fig. 13).

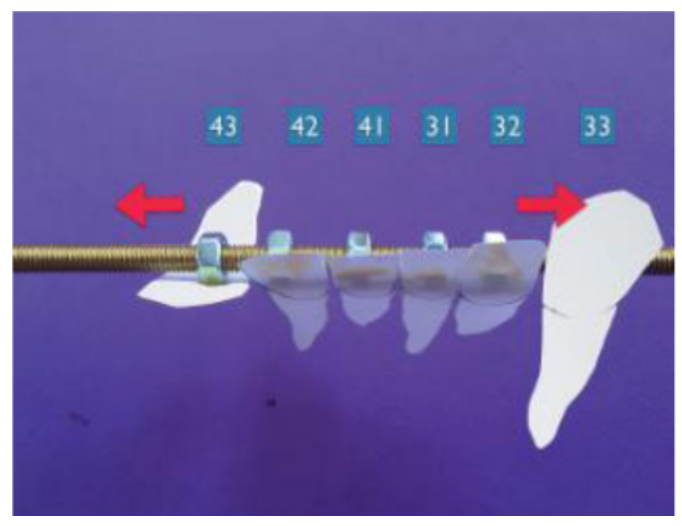

Figure 13

Paper teeth glued on screws forming the bonded studs. The screws are threaded on a rod modeled on the twisted wire. The screw can move because it is not glued to the wire, like a stud that would no longer be glued to the twisted wire. The two canines turn in opposite directions, both "deviating"

by moving toward the end of the wire. 
The posterior-anterior force projects the incisors and canines forward. Anterior projection of the frontal teeth induces diastema opening between incisors and canines.

Let us see how each of the preceding observations is consistent with the theory:

If at the same time, the adhesive breaks away from the adhesive-wire junction and the application of an external force (disto-mesial), the tooth is rotated around the wire.

Observation 1: The wire keeps its original shape.

The movement observed on the teeth does not involve wire deformation contrary to the previously developed hypothesis of the active wire.

Observation 2: The affected teeth are moved with a high intensity. The roots can come out of the cortex.

This phenomenon is not natural, and it does not occur in the absence of compression wire bonded to the teeth.

This leads us to a quantification of the movements:

Considering a 0.0195" 6-strand twisted coaxial wire (5 strands wound around a central wire), there are five turns required to twist it back to the same strand. However, five turns on this type of wire correspond to a distance of $1.8 \mathrm{~mm}$ (Fig. 14).

If a tooth moves in translation of 1.8 $\mathrm{mm}$, this corresponds to a complete revolution around the wire, i.e., a rotation of $360^{\circ}$.

In photos of observed cases, we observe rotations of $1030^{\circ}$, which are consistent with space apertures

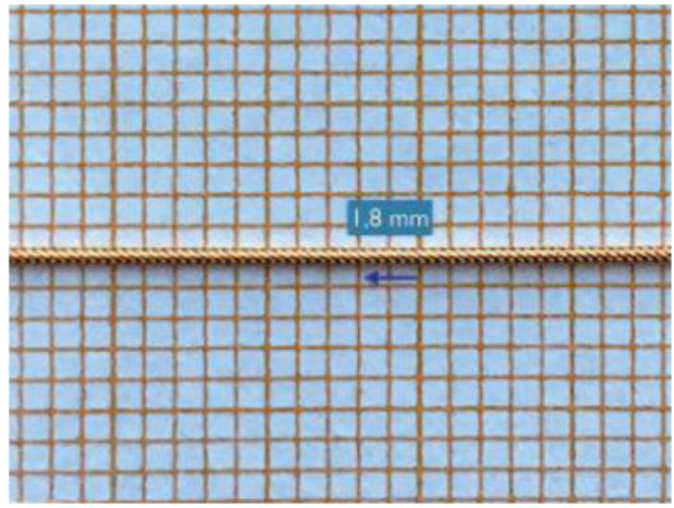

Figure 14

Five turns of a 6-strand coaxial wire corresponds to a wire length of $1.8 \mathrm{~mm}$. Because a tooth moves in translation on this strand, it must rotate by $360^{\circ}$. The rotations of $10-30^{\circ}$ observable on the photographic documents correspond to lateral movements of $0.05-0.15 \mathrm{~mm}$.

of $0.05-0.15 \mathrm{~mm}$. These small space apertures are hard to see in the photos.

Our hypothesis remains valid because $30^{\circ}$ is an extremely strong rotation for a reduced movement in translation.

Observation 3: This movement stops immediately after the wire is removed.

The posterior-anterior force does not stop, but the rotational movement guided by the wire disappears. The twisted teeth are at least partially straightened-if the occlusion does not interfere-in all cases in which we have had the opportunity to observe the patient in the months following wire removal.

Observation 4: The anterior joint is sometimes disturbed with an incisive occlusion end to end up to the reversal of the anterior joint. 
An anterior projection is observed, so the observation is consistent with the hypothesis of the presence of a posterior-anterior or disto-mesial force.

Observation 5: The rotational direction observed on the turned canines is always opposite to the winding direction of the strands.

This is the most important observation that undermines all the hypotheses of "active wire." There is therefore a necessary link between the rotation direction of the tooth and the winding direction of the strands of the twisted wire.
Observation 6: "Wire syndrome" often occurs several years after wire bonding, but its evolution is rapid over the following few months.

This observation corroborates the fact that the phenomenon is linked to the failure of the adhesive-wire junction, which can be caused by ultrasonic vibrations during a descaling session or by the simple wear and tear of this junction. These cases of "wire syndrome," due to their late manifestation, are difficult to explain using the "active wire" theory.

\section{CLINICAL IMPLICATIONS OF THESE OBSERVATIONS}

\section{Reinforcement of the adhesive- wire bonding}

It is important to reinforce the adhesive-wire bonding as much as possible.

When handling the wire, to best adapt to the lingual surfaces, the operator must wear gloves to avoid contaminating the wire.

We propose degreasing the wire with acetone then applying a "metal primer," which will chemically increase the adhesion before attaching the adhesive studs.

Finally, adhesion can be mechanically improved by sandblasting the parts of the wire that are intended to be positioned under the studs.

The choice of wire is also important in the strengthening of the bond. Paolone et al. ${ }^{13}$ showed that 6 -strand coaxial twisted wires are more retentive than 3-strand wires and that these same wires, if gold plated, have the highest values in tensile tests when evaluating wire/composite adhesion.

\section{Steps to follow on discovery of the syndrome}

When "wire syndrome" is detected, wire removal should be planned (Fig. 15).

Sometimes, we may be confronted by a patient-especially if they have been transferred from another practice-who is reluctant to have the wire removed, fearing a need to repeat the treatment. It is therefore important to state, unequivocally, the toxicity of the wire if it is left in place.

If the situation is not too worrying, we recommend a monitoring period of a few months, without any new restraint, to allow natural resolution of this unwanted movement.

On the other hand, if "wire syndrome" is very pronounced and the 

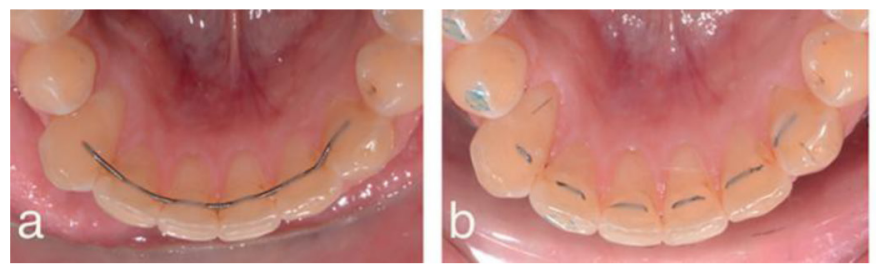

Figure 15

a) Discovery of "wire syndrome." b) In an urgent same day appointment, the wire is simply cut and polished. c) Complete removal of the adhesive and wire during an appointment scheduled 3 months later; this allows practitioners to control the beginning of the "natural" ending of this unwanted movement on 43.

tooth is mobile, orthodontic treatment to replace the root in a more favorable bone environment should start immediately after wire removal².

\section{Patient information}

All patients receiving one or two bonded retention wires should be informed of the need for their orthodontist to monitor them. While specifying the very exceptional character of these

\section{CONCLUSION}

"Wire syndrome," or movement of the teeth connected by a bonded wire of twisted contention in steel, is an undesirable consequence of this type of fixed retention. It is rare, but its consequences can be dramatic for both dental and periodontal health.

We present two theories to explain these movements:

The first is that the wire is active during bonding or activated by the interposition of a hard foreign body during rebonding.

The second is that if the adhesive breaks away from the adhesive-wire junction and if an external force is situations, in order not to trigger an anxious reaction, it is recommended to explain to patients that the teeth can move and that it is imperative to consult if any movement is observed. It is important to clarify that these movements can occur a long time after the retention wire is in place. A simple photograph of the affected arch, sent by email to the office, can suffice to inform the patient about the degree of the urgency for making an appointment.

applied at the same time, the tooth is rotated around the wire.

The wires must absolutely be passive, clean, and rebonded precisely if necessary. Rebonding must be performed with the same care as our orthodontic treatments.

With a good knowledge of the different forms of "wire syndrome," it is possible to detect the beginning of the process very early in a routine checkup and then remove the wire. Our correspondents, general practitioners, and periodontists must therefore be informed to alert the patient in case of doubt. 


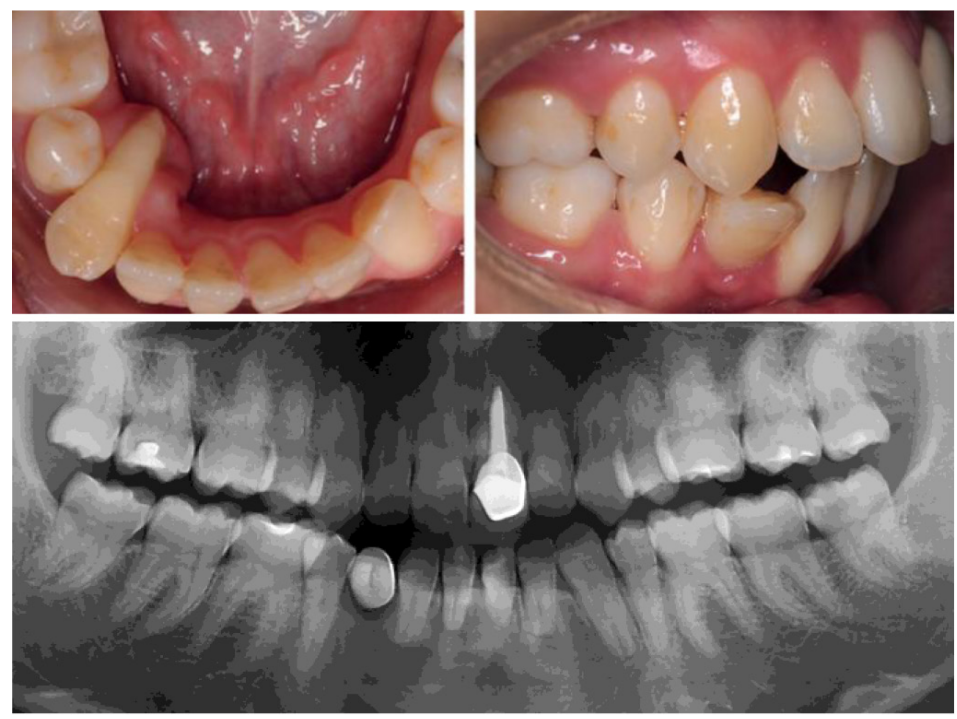

Figure 16

Extreme case of "wire syndrome." The canine is out of the cortex almost to the apex. The patient was referred for endodontic treatment of 43 because it no longer responded to the vitality test. 42 and 41 were still vital. An orthodontic correction will be attempted.

If it is understood that the wire should be removed immediately, when should we treat the periodontium and when should orthodontic treatment be resumed?

The third part of this triptych will be devoted to the periodontal consequences of "wire syndrome" and to proposals for periodontal treatment and orthodontic retreatment timing even in the most extreme cases (Fig. 16).

\section{Conflict of interest}

The authors declare that they have no conflict of interest.

\section{BIBLIOGRAPHY}

1. Djeredou KB, Thiam A, Pesson DM, Haidara-Ntagry F, Toure S. Fiabilité des empreintes à I'alginate de Classe A. Odonto-Stomatologie Tropicale 2001;95: 29-32.

2. Jacobs $\mathrm{C}$. Single tooth torque correction in the lower frontal area by a completely customised lingual appliance. Head Face Med 2017;13(1):18.

3. Kučera J, Marek I. Unexpected complications associated with mandibular fixed retainers: A retrospective study. Am J Orthod Dentofacial Orthop 2016;149(2):202-211.

4. Kiliaridis S, Johansson A, Haraldson T, Omar R, Carlsson GE. Craniofacial morphology, occlusal traits, and bite force in persons with advanced occlusal tooth wear. Am J Orthod Dentofacial Orthop 1995;107:286-92.

5. Muller C, Hitmi L, Roussarie F, Attal JP. Méthode simple et rapide de contention indirecte. Orthod Fr 2009;80:233-238. 
6. Palanisami P, Narasimhan TM, Fernandez JE. The effect of sitting on peak pinch strength, in Aghazadeh F. (Ed.), Advances in Industrial ergonomics and safety VI, Taylor and Francis, London. 1994:587-94.

7. Paolone MG, et al. Tensile test and interface retention forces between wires ans composite in lingual fixed retainers. International orthodontics 2015;13:210-220.

8. Reitan K. Clinical and histologic observations on tooth movement during and after orthodontic treatment. Am J Orthod 1967;53(1):721-745.

9. Roussarie F, Benkimoun F. Collage en vision directe d'une contention indirecte. L'Orthodontiste 2014;3:14- 18.

10. Roussarie F, Douady G, Effets indésirables des fils de contention collés : le " syndrome du fil " 1re partie, Rev Orthop Dentofaciale 2015;49(4):411-426.

11. Schmidt RT, Toews JV. Grip strenght as measured by the jamardynanometer. Arch Phys Med Rehabil 1970;51(6):321-7.

12. Sifakakis I, Pandis N Eliades, Makou TM, M Katsaros C, Bourauelf C. In-vitro assessment of the forces generated by lingual fixed retainers. Am J Orthod Dentofacial Orthop 2011;139:44-8.

13. Wolf $\mathrm{M}$, et al. Post-treatment changes in permanent retention. J Orofac Orthop 2016;77(6):446-453.

14. Zachrisson BU. Important aspects of long-term stability. J Clin Orthod 1997;31:562-83. 